INPLASY

PROTOCOL

To cite: Yu et al. Nucleic acid amplification techniques for rapid diagnosis of nontuberculous mycobacteria: A protocol of systematic review and meta-analysis. Inplasy protocol 2020110076. doi: 10.37766/inplasy2020.11.0076

Received: 18 November 2020

Published: 18 November 2020

Corresponding author:

Lihua Lin

IlhzI0126@163.com

Author Affiliation: Zhejiang Chinese Medicine and Western Medicine Integrated Hospital

Support: None.

Review Stage at time of this submission: The review has not yet started.

Conflicts of interest:

None.

\section{Nucleic acid amplification techniques for rapid diagnosis of non-tuberculous mycobacteria: A protocol of systematic review and meta-analysis}

Yu, G1; Shen, Y2; Xu, X3; Lin, L4.

Review question / Objective: Nontuberculous mycobacteria (NTM) infection is similar to Mycobacterium tuberculosis (MTB) infection. Early clinical identification and differentiation of NTM and MTB infections continues to be a major challenge. Nucleic acid amplification tests (NAATs) have the ability to efficiently and rapidly detect pathogens and are widely used in mycobacterial infections. The purpose of this study was to evaluate the comprehensive evidence for the use of NAATs for rapid NTM diagnosis.

INPLASY registration number: This protocol was registered with the International Platform of Registered Systematic Review and Meta-Analysis Protocols (INPLASY) on 18 November 2020 and was last updated on 18 November 2020 (registration number INPLASY2020110076).

\section{INTRODUCTION}

Review question / Objective: Nontuberculous mycobacteria (NTM) infection is similar to Mycobacterium tuberculosis (MTB) infection. Early clinical identification and differentiation of NTM and MTB infections continues to be a major challenge. Nucleic acid amplification tests (NAATs) have the ability to efficiently and rapidly detect pathogens and are widely used in mycobacterial infections. The purpose of this study was to evaluate the comprehensive evidence for the use of NAATs for rapid NTM diagnosis.

Rationale: Nucleic acid amplification tests (NAATs) have the ability to efficiently and rapidly detect pathogens and are widely used in mycobacterial infections. 
Condition being studied: NTM can also cause a number of infections, of which pulmonary infections are the most common, accounting for $65-90 \%$. The treatment options for infections caused by NTM vary between species, which requires identification of the NTM species. Conventional diagnosis requires waiting for the results of Mycobacterium culture before species identification, and Mycobacterium culture takes several weeks and sensitivity is still at low side. On the other hand, the clinical symptoms of NTM and MTB infections are very similar, and NTM infections can easily be misdiagnosed as MTB infections, resulting in inappropriate treatment and delayed treatment. Early clinical identification and differentiation of NTM and MTB infections continues to be a major challenge Therefore, rapid diagnosis of NTM and differentiation of NTM species is essential in the management of NTM infection. Nucleic acid amplification tests (NAATs) have the ability to efficiently and rapidly detect pathogens and are widely used in mycobacterial infections. Studies have shown that NAATs also have good diagnostic efficacy in the early diagnosis of NTM. However, there is still no systematic review and meta-analysis for NAATs to diagnose NTM. The purpose of this study was to evaluate the comprehensive evidence for the use of NAATs for rapid NTM diagnosis.

\section{METHODS}

Search strategy: Search strategy of PubMed will be listed as follows: \#1 "Nontuberculous Mycobacteria"[Mesh] OR "Non-Tuberculous Mycobacteria" OR "Atypical Mycobacterium" OR "Nontuberculous Mycobacterium" OR "Mycobacteria, Atypical" OR "Tuberculoid Bacillus" OR "Mycobacterium, Atypical" OR "Atypical Mycobacteria" OR "M ycobacterium terrae" OR "Mycobacterium duvalii" OR "Mycobacterium obuense" OR "Mycobacterium gilvum" OR "Mycobacterium gordonae" OR "Mycobacterium szulgai" OR "Mycobacterium flavescens" \#2
"Mycobacterium"[Mesh] OR Mycobacteria \#3 \#1 OR \#2 \#4 "Nucleic Acid Amplification Techniques"[Mesh] OR "Polymerase Chain Reaction"[Mesh] OR "Real-Time Polymerase Chain Reaction"[Mesh] OR "Reverse Transcriptase Polymerase Chain Reaction"[Mesh] OR "Multiplex Polymerase Chain Reaction"[Mesh] \#5 \#3 AND \#4 The other two databases will use a similar search strategy.

Participant or population: Participants with suspected NTM infection, who are confirmed the diagnosis by NAATs. Participants of any ethnicity, sex, or age will be included.

Intervention: NAATs will be identified as index tests.

Comparator: Single-arm studies will also be included if participants, index tests, outcomes meet the inclusion criteria. Comparator tests are not a mandatory indicator.

Study designs to be included: Any type of study design such as retrospective studies, prospective studies, case-control studies, if the study had assessed the efficacy of NAATs in diagnosing NTM. Studies that report only sensitivity or specificity will be excluded.

Eligibility criteria: Original studies that diagnosed NTM by NAATs and have access to the full text will be included in this systematic review and meta-analysis. Clear reference standards in the original study. True positive (TP), false positive (FP), false negative (FN), and true negative (TN) values for the index tests could be extracted directly or calculated from the original studies. Studies reported in languages other than English, Studies with less than 10 specimens, conference abstracts without full articles, and case reports will be excluded.

Information sources: We will search candidate studies that assessing the accuracy of NAATs for diagnosis of NTM through PubMed, Embase and the Cochrane Library until November 2020. 
References cited in a review or metaanalysis will also be evaluated to identify additional studies.

Main outcome(s): The sensitivity and specificity of the index tests will be considered as the main outcome. Sensitivity is the probability that the index tests will detect positive in an infected patient. Specificity is the probability that the index tests will detect negative in a noninfected patient.

Quality assessment / Risk of bias analysis: Following a revised tool for Quality Assessment of Diagnostic Accuracy Studies (QUADAS-2), two researchers will independently evaluate the study quality. The discrepancy will be handled in the same way as in the data extraction phase. publication bias assessment is not required by the PRISMA-DTA statement. The Grading of Recommendations Assessment, Development and Evaluation (GRADE) guideline will be used to assess the strength of the body of evidence.

Strategy of data synthesis: TP, FP, FN, and TN values for the index tests in each included study will be obtained first, then the estimated pooled sensitivity and specificity of NAAT associated with the 95\% confidence interval (CI) will be calculated using MedCalc Statistical Software version 15.2.2 (MedCalc Software bvba, Ostend, Belgium; http:// www.medcalc.org; 2015). Forest plots for sensitivity and specificity will be generated for each study. At least 4 eligible studies will be required to perform the metaanalysis and meta-regression analyses for predefined variable parameters using Stata version 15.0 (Stata Corp., College Station, TX, USA) with the midas command packages.

Subgroup analysis: The heterogeneity will be determined by using the 12 statistic. $12=$ $0 \%$ will indicate no observed heterogeneity, $12<50 \%$ will be considered minor heterogeneity, $12>50 \%$ will be considered substantial heterogeneity. When significant heterogeneity is observed, subgroup, metaregression and sensitivity analyses will be used to explore sources of heterogeneity. Subgroup and meta-regression analyses will perform on different types of study design; patient selection method; index test types; specimen types; specimen condition; NTM types and some test procedures (e.g., homogenization).

Sensibility analysis: When significant heterogeneity is observed, sensitivity analyses will be used to explore sources of heterogeneity.

Language: No restriction.

Country(ies) involved: China.

Keywords: Nontuberculous mycobacteria, diagnostic accuracy, nucleic acid amplification test, Polymerase Chain Reaction, meta-analysis.

Contributions of each author:

Author 1 - Guocan Yu - The author searched databases, selected literatures, managed data, assessed quality, drafted and revised the manuscript.

Email: dabaitwo@163.com

Author 2 - Yanqin Shen - The author searched databases, selected literatures, managed data and evaluated quality.

Email: yanqinshen@yeah.net

Author 3 - Xudong Xu - The author drafted and revised the manuscript.

Email: xuxudong234@163.com

Author 4 - Lihua Lin - The author provided statistical expertise, read, feedback and approved the final manuscript.

Email: Ilhz10126@163.com 